\title{
miR-30 decreases multidrug resistance in human gastric cancer cells by modulating cell autophagy
}

\author{
XINMING DU ${ }^{1,2}$, BING LIU ${ }^{3}$, XUERONG LUAN ${ }^{2}$, QING CUI ${ }^{2}$ and LEPING LI ${ }^{1}$ \\ ${ }^{1}$ Department of Gastrointestinal Surgery, Shandong Provincial Hospital Affiliated to Shandong University, Jinan, \\ Shandong 250021; ${ }^{2}$ Department of Gastrointestinal Surgery, Zibo Central Hospital, Zibo, Shandong 250020; \\ ${ }^{3}$ Department of Vascular Surgery, Affiliated Hospital of Qingdao University, Qingdao, Shandong 266071, P.R. China
}

Received June 6, 2017; Accepted October 20, 2017

DOI: $10.3892 /$ etm.2017.5354

\begin{abstract}
Chemotherapy is an important treatment modality for gastric cancer, and multidrug resistance (MDR) represents a major obstacle for successful cancer chemotherapy. There is a lack of research on whether microRNA (miR)-30a regulation affects the chemosensitivity of resistant gastric cancer cells, and mechanisms underlying the effects of miR-30a on drug resistance and cell autophagy require further investigation. In the present study, the expression of miR-30a and its effects in cisplatin (CDDP)-resistant human gastric cancer cells were investigated. A CDDP-resistant variant of the SGC-7901 cell line (SGC-7901/CDDP) was established by exposing the cells to gradually increasing drug concentrations, and miR-30a expression was detected by reverse transcription-semi quantitative polymerase chain reaction (RT-sqPCR). To examine the effect of miR-30a expression in the SGC-7901/CDDP cells, miR30a mimics or negative control miRNA were transfected into the cells, and a Cell Counting Kit- 8 assay was performed to analyze the chemosensitivity of the different cell groups. RT-sqPCR and western blot analysis were also used to measure MDR 1 mRNA and P-glycoprotein expression, and the light chain (LC)3-II/LC3-I ratio. Furthermore, apoptosis induced by the chemotherapeutic CDDP in the different groups was assessed using flow cytometry. The results demonstrated that low expression of miR-30a was associated with chemoresistance in gastric cancer cells, and in the chemoresistant cell line SGC7901/CDDP, CDDP-induced apoptosis was weakened. Additionally, it was demonstrated that the LC3-II/LC3-I ratio was elevated in SGC7901/CDDP cells compared with chemosensitive SGC7901 cells $(\mathrm{P}<0.001)$, which could be attenuated by upregulating $\mathrm{miR}-30 \mathrm{a}$ expression $(\mathrm{P}<0.001 \mathrm{vs}$.
\end{abstract}

Correspondence to: Dr Leping Li, Department of Gastrointestinal Surgery, Shandong Provincial Hospital Affiliated to Shandong University, 324 Jingwuweiqi Road, Jinan, Shandong 250021, P.R. China

E-mail: lileping@medmail.com.cn

Key words: miR-30a, drug resistance, gastric cancer, autophagy
SGC7901/CDDP control cells). These results suggested that autophagy may contribute to drug resistance in gastric cancer cells, and that the reduction of LC3-II in response to miR-30a overexpression may inhibit chemoresistance-associated autophagy in gastric cancer cells.

\section{Introduction}

Gastric cancer is the fourth most common cancer and second leading cause of cancer-related deaths worldwide $(1,2)$. Although surgery is the most effective treatment modality for patients with resectable tumors, due to the low rates of early detection and diagnosis, many patients with gastric cancer in China are diagnosed at an advanced and unresectable clinical stage. For advanced gastric cancer and for patients with recurrent cancer who are not suitable candidates for reoperation, chemotherapy remains the preferred treatment method (3). However, at present, intrinsic or acquired multidrug resistance (MDR) represents a major obstacle for successful cancer chemotherapy (4). The mechanism underlying MDR is complex due to interactions between various factors, including drug efflux, the alteration of drug targets, DNA damage repair, cell proliferation and apoptosis, and cell cycle changes (5-7).

Noncoding RNAs (ncRNAs) have drawn much attention in the field of cancer research. Of particular interest are microRNAs (miRNA/miRs), which are small ncRNAs that are able to post-transcriptionally modulate gene expression, with the primary functions of controlling physiological and pathological processes, including cancer onset, growth, and progression (8). To date, $>200$ miRNAs have been found to be associated with gastric cancer development, progression, and therapeutic response, and numerous studies have indicated that miRNAs may play an important role in the MDR of various types of cancer, including gastric cancer (9-11). It has previously been reported that miR-30a is significantly downregulated in gastric cancer cells $(12,13)$. However, there is a lack of research on whether miR-30a regulation can affect the chemosensitivity of resistant gastric cancer cells, and the underlying mechanisms regarding the potential effects of miR-30a on drug resistance and cell autophagy require further investigation. In the present study, we assessed the potential effect of miR-30a on the MDR of the SGC7901 gastric cancer cell line. Additionally, we aimed to provide insight into the 
molecular mechanism underlying the effect of miR-30a on MDR, with focus on chemotherapy-induced autophagy.

\section{Materials and methods}

Cell culture. The human gastric cancer cell line SGC-7901 was purchased from the Cell Bank of the Chinese Academy of Medical Sciences (Shanghai, China) and maintained in RPMI-1640 medium containing 10\% FBS (both from Gibco; Thermo Fisher Scientific, Inc., Waltham, MA, USA) and $0.1 \%$ penicillin-streptomycin (Beijing Solarbio Science \& Technology Co., Ltd., Beijing, China) at $37^{\circ} \mathrm{C}$ in a humid atmosphere (5\% $\mathrm{CO}_{2}, 95 \%$ air).

Cisplatin (CDDP) was purchased from Qilu Pharmaceutical Co., Ltd. (Jinan, China) and dissolved with normal saline before use. A CDDP-resistant variant SGC-7901/CDDP cell line was established by gradually increasing the drug concentration $(0.1,0.2,0.3,0.4,0.5$, and $0.6 \mu \mathrm{g} / \mathrm{ml}$ CDDP each for two weeks). The SGC-7901/CDDP phenotype was then maintained by supplementing the cell medium with $0.6 \mu \mathrm{g} / \mathrm{ml}$ cisplatin, which was stopped 2 weeks prior to experiments.

Cell transfection. miR-30a mimics, and scramble miRNA negative control (NC miRNA) were synthesized by Shanghai GenePharma Co., Ltd. (Shanghai, China). SGC-7901/CDDP cells were transfected with $100 \mathrm{nM}$ miR-30a mimics for miR-30a overexpression. Transfection with NC miRNA was performed in parallel as a control. Transfection was performed using Lipofectamine 2000 reagent (Invitrogen; Thermo Fisher Scientific, Inc., Waltham, MA, USA) according to the manufacturer's protocol (13). The transfection efficiency was measured via the reverse transcription (RT)-PCR analysis of miR-30a expression described below.

Reverse transcription-semi-quantitative polymerase chain reaction (RT-sqPCR) analysis of miR-30a and MDR1 mRNA expression. A TRIzol kit was used to extract total RNA according to the manufacturer's instructions (Invitrogen; Thermo Fisher Scientific, Inc.), and the RNA samples were quantified by UV spectrophotometry.

miRNA-specific cDNA was obtained by RT using stem-loop primers and a TaqMan MicroRNA Reverse Transcription kit (Applied Biosystems; Thermo Fisher Scientific, Inc.), the RT primer used for miR-30a was 5'-CGTCGCTACATCCAGTGT AGCATATGCGACGCTTCCAGT-3'. Subsequently, the level of mature miR-30a was detected using a TaqMan MicroRNA Assay kit (Applied Biosystems; Thermo Fisher Scientific, Inc.) according to the manufacturer's instructions. The PCR primers used indluded: miR-30a forward, 5'-TGTAAACATCCTCGA C-3' and reverse, 5'-ACATCCAGTGTAGCATA-3'; U6 forward, 5'-CTTCGGCAGCACATATACTAAAAT-3' and reverse, 5'-CAGGGGCCATGCTAAATCTTC-3'. The PCR reaction was performed for $35 \mathrm{cycles}$ at $94^{\circ} \mathrm{C}$ for $30 \mathrm{sec}, 50^{\circ} \mathrm{C}$ for $30 \mathrm{sec}$ and $72^{\circ} \mathrm{C}$ for $30 \mathrm{~min}$.

To measure the expression of MDRI mRNA, RT was carried out with $2 \mu \mathrm{g}$ extracted total RNA using a First Strand cDNA Synthesis kit (Toyobo Life Science, Osaka, Japan). For semi-quantitative RT-PCR, 100 ng cDNA was amplified with gene-specific primers using Taq DNA polymerase in 10X PCR buffer, $4 \mathrm{X}$ dNTP mixture and $\mathrm{MgCl}_{2}$. The following primers (Shanghai GeneCore BioTechnologies Co., Ltd., Shanghai, China) were used for the specific amplification of MDRI forward, 5'-AGACATGACCAGGTATGCCTAT-3' and reverse, 5'-AGCCTATCTCCTGTCGCATTA-3'. The expression of GAPDH (forward, 5'-GAGGGGCCATCCACAGTCTT-3' and reverse, 5'-TTCATTGACCTCAACTACAT-3') was used as an internal control. The PCR reaction was performed for 35 cycles at $94^{\circ} \mathrm{C}$ for $30 \mathrm{sec}, 56^{\circ} \mathrm{C}$ for $30 \mathrm{sec}$ and $65^{\circ} \mathrm{C}$ for $1 \mathrm{~min}$.

The PCR products were separated on $2 \%$ agarose gels containing $0.5 \mu \mathrm{g} / \mathrm{ml}$ ethidium bromide and photographed under a UV transilluminator, and AlphaEaseFC software was used to analyze the relative light intensities. Three independent experiments with triplicate samples were performed.

Detection of cell chemosensitivity to CDDP. Cell chemosensitivity was detected in the following four cell groups: SGC7901 (chemosensitive) cells, SGC7901/CDDP (chemoresistant) control cells, SGC7901-NC miRNA cells (transfected with NC miRNA), and SGC7901/CDDP-miR30a mimics cells (transfected with miR30a mimics). The cells of the different groups were seeded in 96 -well plates $\left(5 \times 10^{3}\right.$ cells/well $)$ and incubated at $37^{\circ} \mathrm{C}$ in a humidified $5 \% \mathrm{CO}_{2}$ atmosphere for $24 \mathrm{~h}$. Subsequently, CDDP was added at final concentrations of $0.02,0.1,0.5,2.5$, 12.5 , and $62.5 \mu \mathrm{g} / \mathrm{ml}$ to the culture medium or an equal volume of vehicle was added as control treatment. At $48 \mathrm{~h}$ after CDDP administration, cell viability was assessed using a CCK-8 assay (Toyobo Life Science) according to the manufacturer's instructions. The absorbance at $450 \mathrm{~nm}$ was measured, from which the cell growth inhibition rate and half maximal inhibitory concentration $\left(\mathrm{IC}_{50}\right)$ of $\mathrm{CDDP}$ was calculated. Additionally, the resistance index of the SGC7901/CDDP cells was calculated as: $\mathrm{IC}_{50}$ of sensitive cell line $/ \mathrm{IC}_{50}$ of resistant cell line. Three independent experiments were performed in triplicate.

Cell apoptosis measurement by flow cytometry. Cell apoptosis was measured using an Annexin V-propidium iodide (PI) apoptosis detection kit (BD556547 ${ }^{\mathrm{TM}}$; BD Pharmingen; BD Biosciences, Franklin Lakes, USA). Cell apoptosis was analyzed in the following cell groups: SGC7901 sensitive cells, SGC7901/CDDP resistant control cells, SGC7901-NC miRNA cells and SGC7901/CDDP-miR30a mimics cells. Following treatment with $5 \mu \mathrm{g} / \mathrm{ml}$ CDDP for $24 \mathrm{~h}$, the cells in the different groups were collected and washed twice with precooled PBS, and then re-suspended in $400 \mu 1$ of Annexin V binding buffer. Subsequently, the cells were incubated with fluorescein isothiocyanate (FITC)-Annexin $\mathrm{V}(5 \mu \mathrm{l})$ for $15 \mathrm{~min}$ at room temperature in the dark, and then with PI $(5 \mu \mathrm{l})$ for $5 \mathrm{~min}$ at $4^{\circ} \mathrm{C}$ in the dark prior to analysis by flow cytometry.

Western blotting. Total protein was extracted from the cells in each group and protein concentration was measured using the bicinchoninic acid method. The protein samples were subjected to SDS-PAGE and transferred to PVDF membranes (EMD Millipore, Billerica, MA, USA) for $2 \mathrm{~h}$. After washing, Tris-buffered saline with Tween solution containing 5\% skimmed milk powder was used to block the membranes for $1 \mathrm{~h}$. Primary antibodies diluted to the appropriate concentrations were incubated with the membranes overnight at $4^{\circ} \mathrm{C}$ : Rabbit anti-LC3A/B (\#12741, 1:1,000; Cell Signaling 
Table I. Cytotoxicity of CDDP in different groups.

SGC7901/CDDP

\begin{tabular}{lccrr}
\cline { 3 - 4 } Variable & SGC7901 & SGC7901/CDDP & NC miRNA & miR-30a mimics \\
\hline $\mathrm{IC}_{50}(\mu \mathrm{M})$ & 1.99 & $6.39^{\mathrm{a}}$ & 7.74 & $2.57^{\mathrm{b}}$ \\
$\mathrm{IR}_{\max }(\%)$ & $83.27 \pm 2.03$ & $68.76 \pm 4.68$ & $67.42 \pm 5.96$ & $74.66 \pm 3.10$ \\
\hline
\end{tabular}

$\mathrm{IR}_{\max }$, the maximum inhibition rate. ${ }^{\mathrm{a}} \mathrm{P}<0.01 \mathrm{vs.} \mathrm{SGC7901;}{ }^{\mathrm{b}} \mathrm{P}<0.01$ vs. SGC7901/CDDP. CDDP, cisplatin.

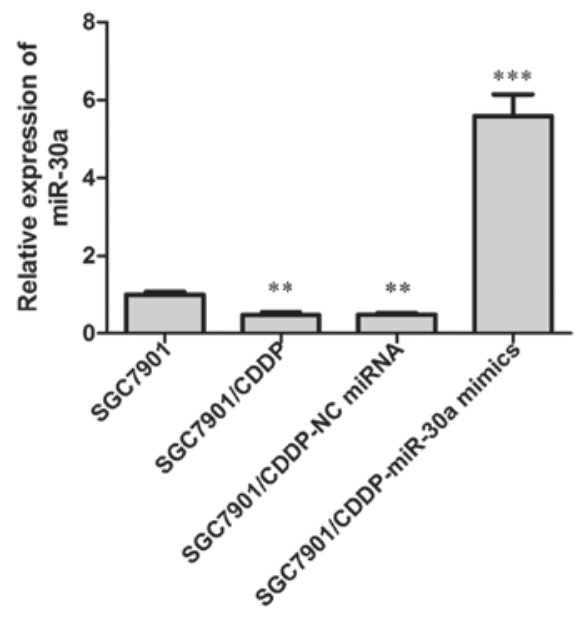

Figure 1. Quantification of miR-30a expression in different cell groups by RT-PCR analysis. miR-30a expression was decreased in chemoresistant SGC-7901/CDDP cells compared with chemosensitive SGC-7901 cells. When transfected with miR-30a mimics or NC miRNA, miR-30a expression in the SGC7901/CDDP cells was notably increased or decreased, respectively. ${ }^{* * *} \mathrm{P}<0.01$ vs. SGC7901; ${ }^{* * *} \mathrm{P}<0.001$ vs. SGC7901/CDDP. CDDP, cisplatin.

Technology, Danvers, MA, USA), mouse anti-Mdr-1 (sc-13131, 1:800; Santa Cruz Biotechnology, Inc., Dallas, TX, USA), and rabbit anti- $\beta$-actin (ab8227, 1:5,000; Abcam, Cambridge, UK) as internal control. The membranes were then washed and incubated with secondary antibody (goat anti-rabbit, ZB-2301, 1:5,000; goat anti-mouse, ZDR5307) (both purchased from ZSGB-Bio, Beijing, China) for $1 \mathrm{~h}$ at room temperature. After washing, ECL substrate (EMD Millipore) was added and the protein bands were analyzed with a gel electrophoresis image analyzer (FR980; Shanghai Furi Science \& Technology Co., Ltd., Shanghai, China).

Statistical analysis. SPSS 18.0 software (SPSS Inc., Chicago, IL, USA) was used to analyze the data. All data were expressed as the mean $\pm \mathrm{SD}$. One-way analysis of variance was used for comparisons among three or more groups, and the post hoc LSD test was used for subsequent comparisons between two groups. $\mathrm{P}<0.05$ was considered to indicate statistical significance.

\section{Results}

Expression of miR-30a in chemoresistant cells. The expression of miR-30a was examined by RT-PCR analysis. Our results showed that in the chemoresistant SGC7901/CDDP

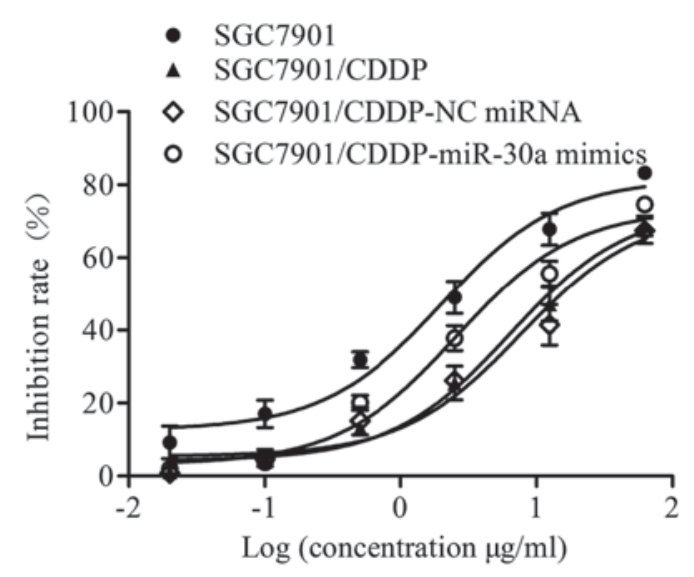

Figure 2. Growth inhibition of cells in different groups. Cells were exposed to increasing concentrations of CDDP or an equal volume of vehicle for up to $48 \mathrm{~h}$. Viable cells were counted using a CCK- 8 kit and denoted as a percentage relative to the untreated controls.

cells, the expression of miR-30a was significantly decreased when compared with that in the chemosensitive SGC7901 cells (Fig. 1; P<0.01). Subsequently, we overexpressed miR-30a endogenous miR-30a expression in the SGC7901/CDDP cells by transfection with miR30a mimics, with NC miRNA transfection performed in parallel, and the transfection efficiency was detected by RT-PCR analysis of miR-30a expression, our data showed that after miR-30a mimics transfection, the miR-30a level in SGC7901/CDDP cells was elevated to about 6-fold of the SGC7901/CDDP control cells.

miR-30a expression is related to gastric cancer cell chemoresistance. To determine the effect of miR-30a on the chemosensitivity of gastric cancer cells, a CCK-8 assay was performed. Our results (Fig. 2 and Table I) showed that the chemosensitivity of SGC7901/CDDP cells was significantly decreased compared with that of SGC7901 cells; notably, the $\mathrm{IC}_{50}$ value of CDDP was elevated from $6.63 \mu \mathrm{M}$ in SGC7901 cells to $21.28 \mu \mathrm{M}$ in SGC7901/CDDP cells. Furthermore, the resistance index of the SGC7901/CDDP cells was 3.21. No significant difference in $\mathrm{IC}_{50}$ was observed between the SGC7901/CDDP $(21.28 \mu \mathrm{M})$ and SGC7901/CDDP-NC miRNA $(25.77 \mu \mathrm{M})$ groups $(\mathrm{P}>0.05)$. By contrast, the $\mathrm{IC}_{50}$ of CDDP in the SGC7901/CDDP-miR-30a mimics group was decreased to $8.56 \mu \mathrm{M}$ ( $\mathrm{P}<0.001$ vs. SGC7901/CDDP group), indicating increased chemosensitivity following miR-30a transfection. 
A
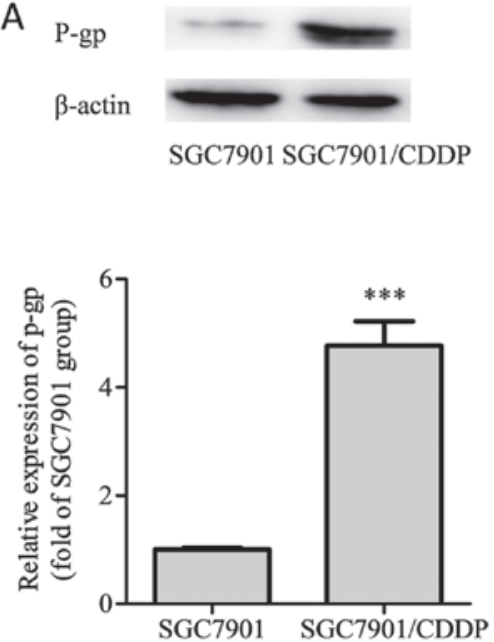

C

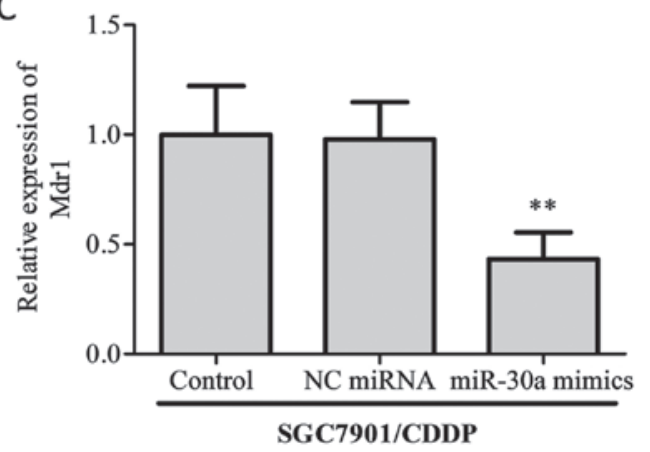

B
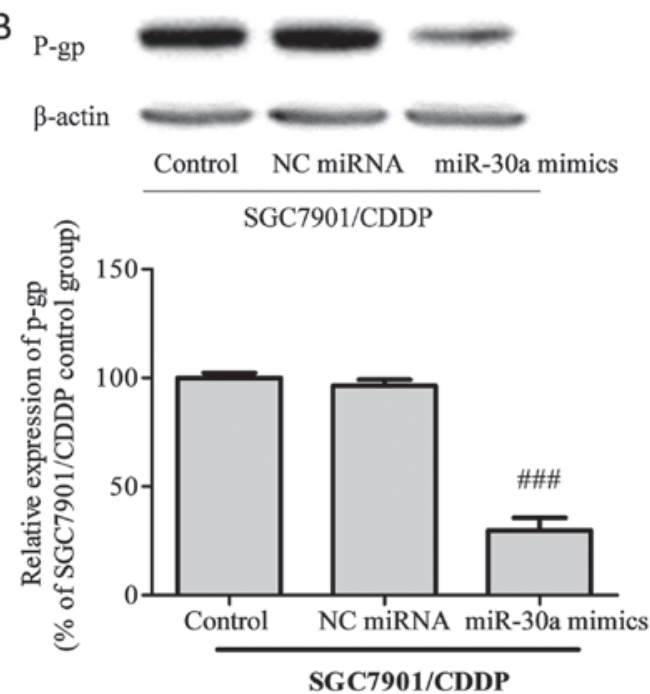

Figure 3. Expression analysis of P-gp and MDR1 in different cell groups. Total protein or RNA was extracted for P-gp and MDR1 analysis. (A) The protein levels of P-gp in SGC7901 and SGC7901/CDDP cells were determined by western blot analysis. (B) P-gp expression was decreased in SGC7901/CDDP cells transfected with miR-30a mimics. (C) The mRNA levels of $M D R 1$ were detected by real time PCR analysis. MDRl mRNA expression was decreased in SGC7901 cells transfected with miR-30a mimics. Control, SGC7901/CDDP cells without transfection treatment; NC miRNA, SGC7901/CDDP cells transfected with NC miRNA; miR-30a mimics, SGC7901/CDDP cells transfected with miR-30a mimics. Data were expressed as the means \pm SD of three independent experiments. ${ }^{* * *} \mathrm{P}<0.001$ vs. SGC7901 group; ${ }^{\# \# \#<0.001 ~ v s . ~ S G C 7901 / C D D P ~ c o n t r o l ~ c e l l s ; ~}{ }^{* *} \mathrm{P}<0.01$ vs. SGC7901/CDDP control group. P-gp, P-glycoprotein; CDDP, cisplatin.

miR-30a regulates MDR-related factors. Subsequently, we examined the protein levels of P-glycoprotein (P-gp) and the mRNA expression of its corresponding gene, $M D R 1$, in SGC7901/CDDP cells. As shown in Fig. 3, the expression of P-gp protein was notably elevated in SGC7901/CDDP cells compared with SGC7901 cells. After transfection with miR-30a mimics, P-gp expression in SGC7901/CDDP cells was significantly decreased. On MDRl mRNA analysis, the same trends as for $\mathrm{P}$-gp protein expression were identified.

miR-30a modulates cell autophagy and increases apoptosis in chemoresistant gastric cancer cells. Cell apoptosis induced by CDDP treatment was analyzed by flow cytometry (Fig. 4A). The total apoptotic rate of SGC7901 cells treated with $5 \mu \mathrm{g} / \mathrm{ml}$ CDDP was $36.5 \%$, while in the SGC7901/CDDP group, the total apoptotic rate was decreased to $17.3 \%$ ( $\mathrm{P}<0.001$ vs. SGC7901 group). Compared with the SGC7901/CDDP group, the total apoptotic rate did not differ significantly in the SGC7901/CDDP-NC miRNA group (19.6\%, P>0.05); however, in the SGC7901/CDDP-miR-30a mimics group, the total apoptotic rate was elevated to $38.9 \%(\mathrm{P}<0.001)$, thus indicating that the upregulation of miR-30a improved chemosensitivity in the SGC7901/CDDP cells.
Light chain (LC)3, which exists as a cytosolic form (LC-I) under normal homeostatic conditions, becomes conjugated to phosphatidylethanolamine (PE) to form the membrane-bound form LC3-II upon autophagy induction (14). Our results showed that in the chemoresistant SGC7901/CDDP cells, the LC3-II/LC3-I ratio was significantly higher than that in the chemosensitive SGC7901 cells (Fig. 4B, P<0.001). Meanwhile, on upregulation of miR-30a in the SGC7901/CDDP cells, the elevated LC3-II/LC3-I ratio was attenuated.

\section{Discussion}

Clinical treatment methods for patients with gastric cancer include surgery, radiation therapy, and chemotherapy. Tumors that develop drug resistance to chemotherapy are a major obstacle for successful cancer chemotherapy. Previously reported mechanisms of chemoresistance include increased drug efflux, mutation of target genes, inactivation of detoxification enzymes, dysfunction of pro-apoptotic proteins and enhancement of DNA repair activity, although the precise mechanisms involved in cancer cell chemoresistance remain to be fully elucidated $(4,15)$. The present study identified 


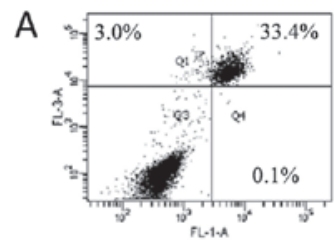

SGC7901

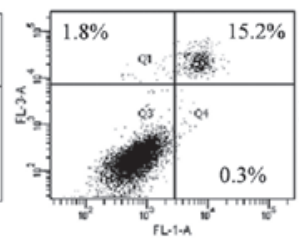

Control

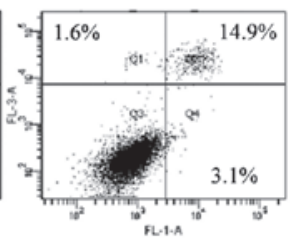

NC MiRNA

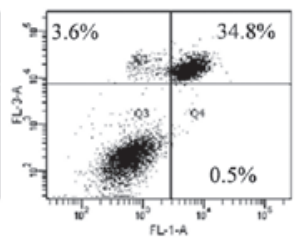

miR-30a mimics

SGC7901/CDDP

B

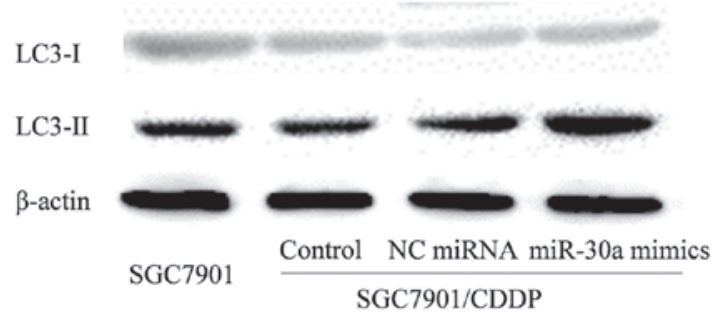

C

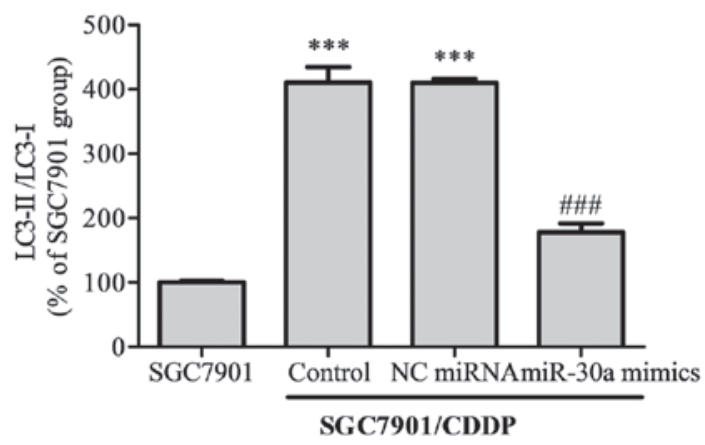

Figure 4. Effects of miR-30a on cell apoptosis and LC3-II/LC3-I expression. (A) Flow cytometric analysis of apoptotic SGC-7901 cells or SGC7901/CDDP cells (control SGC7901/CDDP cells without transfection and SGC7901/CDDP cells transfected with NC siRNA or miR-30a mimics) $24 \mathrm{~h}$ after treatment with $5 \mu \mathrm{g} / \mathrm{ml}$ CDDP. (B) Western blot analysis of LC3-II/LC3-I expression. (C) Relative LC3-II/LC3-I expression determined from density analysis. Data were expressed as the means \pm SD of three independent experiments. Control, SGC7901/CDDP control cells without transfection treatment; NC miRNA, SGC7901/CDDP cells transfected with NC miRNA; miR-30a mimics, SGC7901/CDDP cells transfected with miR-30a mimics. ${ }^{* * *}$ P $<0.001$ vs. SGC7901

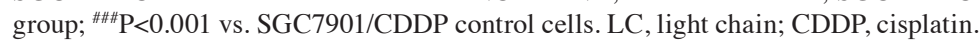

miR-30a as a novel chemosensitivity-associated miRNA related to autophagy and apoptosis.

Increasing evidence has demonstrated that miRNAs are involved in chemoresistance in many tumor types $(8,16,17)$. In this research, we confirmed that miR-30a expression was downregulated in the chemoresistant SGC7901/CDDP cell line. Additionally, our results showed that upregulating miR-30a by transfection with miR-30a mimics could attenuate the chemoresistance of SGC7901/CDDP cells, observed as a decrease in the $\mathrm{IC}_{50}$ of CDDP from 21.28 to $8.56 \mu \mathrm{M}$ (P<0.05, SGC7901/CDDP vs. SGC7901/CDDP-miR-30a mimics group). These results suggest that miR-30a plays an important role in the chemoresistance of gastric cancer cells. P-gp, a $170-k D a$ transmembrane glycoprotein encoded by the human MDRl [ATP-binding cassette (ABC) subfamily $\mathrm{B}$ member 1] gene, is a member of the $\mathrm{ABC}$ transporters family, and serves as a drug efflux pump that extrudes a wide spectrum of chemotherapeutic agents from MDR cancer cells (18). Accordingly, P-gp substrates can generally induce $M D R 1 / \mathrm{P}$-gp expression in MDR cancer cells (19). In this study, we found that the expression level of P-gp in the SGC7901/CDDP cells was significantly higher than that in the SGC7901 cells, suggesting that overexpression of P-gp may be a mechanism by which SGC7901 cells develop drug resistance. Several miRNAs have been identified as critical regulators of P-gp-mediated drug resistance in many cancers $(13,16,17)$. Similarly, we revealed that the expression of P-gp protein and its cognate gene MDRl could be affected by miR-30a expression, indicating that miR-30a may arrest the chemoresistance and growth of SGC7901/CDDP cells by inhibiting P-gp activity and expression. This was consistent with previous research (13).

Apoptosis has been widely investigated in the past few decades and is established as the major mechanism of programmed cell death (PCD) (20). Additionally, apoptosis is believed to be the major type of cell death triggered by chemotherapeutic drugs. However, apoptosis is not the sole route of PCD; both apoptosis ('self-killing') and autophagy ('self-eating') serve a role in self-destructive processes in cells, and interact to execute gastric cancer cell death $(21,22)$. Autophagy is a survival strategy employed by cells experiencing nutrient deprivation or other stresses, which is a tightly regulated by the lysosomal degradation pathway, and is considered to be essential for cell growth, differentiation, development, survival and homeostasis. However, while autophagy is generally considered to be a survival mechanism 
under normal homeostatic conditions, it has both pro-death and pro-survival roles in cancer $(21,23)$.

Chemotherapeutic drugs can induce both apoptosis and autophagy in the treatment of cancer, and it was previously reported that autophagy helps cancer cells evade apoptosis, and thus contributes to chemoresistance (24). Additionally, cancer cells that respond to drugs by inducing autophagy are more drug-resistant (25): For example, in response to 5-fluorouracil (5-FU) and cisplatin, chemosensitive cell lines were found to undergo apoptosis, whereas chemoresistant populations underwent autophagy (25-28). It is believed that autophagy can play both positive and negative roles in promoting apoptosis. Previous research demonstrated the cytoprotective role of autophagy in response to 5-FU treatment in gastric cancer cells $(27,28)$, while a different study showed that 5-FU may suppress miR-30 to upregulate beclin-1 and thus induce autophagic cell death and cell proliferation arrest in gastric cancer cells (29). Nevertheless, many studies have shown that autophagy protects various tumor cells against apoptosis induced by chemotherapeutic drugs $(23,30,31)$.

LC3 is one of the biomarkers of autophagy, and on initiation of autophagy, cytosolic LC3-I is converted into membrane-bound LC3-II, which is an essential step in autophagosome formation, and thus the abundance of LC3-II is correlated with the number of autophagosomes (32). In this research, we confirmed that autophagy was induced while cell apoptosis was mitigated in the SGC7901/CDDP resistant cells after treatment with CDDP, relative to CDDP-treated SGC7901 cells. These findings were consistent with previous reports (30-32).

It has previously been confirmed that the autophagic protein beclin-1 is a putative target of miR-30a (33). To understand the mechanisms of miR-30a in gastric cancer chemoresistance, we examined the effect of miR-30a overexpression on the autophagy status of cells. We found that low expression of miR-30a was related to chemoresistance in gastric cancer cells, and in the chemoresistant cell line SGC7901/CDDP, CDDP-induced apoptosis was weakened. Furthermore, the LC3-II/LC3-I ratio was elevated in the SGC7901/CDDP cells when compared with that in the chemosensitive SGC7901 cells $(\mathrm{P}<0.05)$, while transfection with miR-30a mimics decreased the LC3-II/LC3-I ratio in the SGC7901/CDDP cells ( $<<0.05$ vs. SGC7901/CDDP control cells). These results suggested that autophagy might contribute to chemoresistance in gastric cancer cells, and that the reduction of LC3-II in response to miR-30a upregulation might inhibit chemoresistance-related autophagy in gastric cancer cells.

In summary, as autophagy plays a dual role in tumor promotion and suppression, further investigation is required to determine whether the activation of autophagy leads to the survival or death of cancer cells during chemotherapy. The present study demonstrated that miR-30a was associated with chemoresistance in SGC7901 cells, and that the underlying mechanism involves, at least in part, the modulation of chemoresistance-related autophagy. Understanding the novel functions of miRNAs may allow us to develop promising therapeutic strategies for enhancing the effects of chemotherapy, which may ultimately improve clinical outcomes in the treatment of cancer patients.

\section{References}

1. Xu W, Yang Z and Lu N: Molecular targeted therapy for the treatment of gastric cancer. J Exp Clin Cancer Res 35: 1, 2016.

2. Siegel R, Ma J, Zou Z and Jemal A: Cancer statistics, 2014. CA Cancer J Clin 64: 9-29, 2014

3. Hu Y, Ying M, Huang C, Wei H, Jiang Z, Peng X, Hu J, Du X, Wang B, Lin F, et al: Oncologic outcomes of laparoscopy-assisted gastrectomy for advanced gastric cancer: A large-scale multicenter retrospective cohort study from China. Surg Endosc 28: 2048-2056, 2014.

4. O'Connor R: The pharmacology of cancer resistance. Anticancer Res 27: 1267-1272, 2007.

5. Choi JH, Lim HY, Joo HJ, Kim HS, Yi JW, Kim HC, Cho YK, Kim MW and Lee KB: Expression of multidrug resistance-associated protein1, P-glycoprotein and thymidylate synthase in gastric cancer patients treated with 5-fluorouracil and doxorubicin-based adjuvant chemotherapy after curative resection. Br J Cancer 86: 1578-1585, 2002.

6. Morin PJ: Drug resistance and the microenvironment: Nature and nurture. Drug Resist Updat 6: 169-172, 2003.

7. Lippert TH, Ruoff HJ and Volm M: Intrinsic and acquired drug resistance in malignant tumors. The main reason for therapeutic failure. Arzneimittelforschung 58: 261-264, 2008.

8. Zhang $M$ and Du X: Noncoding RNAs in gastric cancer: Research progress and prospects. World J Gastroenterol 22: 6610-6618, 2016

9. Tan P and Yeoh KG: Genetics and molecular pathogenesis of gastric adenocarcinoma. Gastroenterology 149: 1153-1162.e3, 2015.

10. Song JH and Meltzer SJ: MicroRNAs in pathogenesis, diagnosis, and treatment of gastroesophageal cancers. Gastroenterology 143: 35-47.e2, 2012.

11. Zhang $\mathrm{M}$ and $\mathrm{Du} \mathrm{X}$ : Noncoding RNAs in gastric cancer: Research progress and prospects. World J Gastroenterol 22: 6610-6618, 2016.

12. Liu Z, Chen L, Zhang X, Xu X, Xing H, Zhang Y, Li W, Yu H, Zeng J, Jia J, et al: RUNX3 regulates vimentin expression via miR-30a during epithelial-mesenchymal transition in gastric cancer cells. J Cell Mol Med 18: 610-623, 2014.

13. Li C, Zou J, Zheng G and Chu J: miR-30a decreases multidrug resistance (MDR) of gastric cancer cells. Med Sci Monit 0: 0, 2016.

14. Lai YC, Chuang YC, Chang CP and Yeh TM: Macrophage migration inhibitory factor has a permissive role in concanavalin A-induced cell death of human hepatoma cells through autophagy. Cell Death Dis 6: e2008, 2015.

15. Shi WJ and Gao JB: Molecular mechanisms of chemoresistance in gastric cancer. World J Gastrointest Oncol 8: 673-681, 2016.

16. An Y, Zhang Z, Shang Y, Jiang X, Dong J, Yu P, Nie Y and Zhao Q: miR-23b-3p regulates the chemoresistance of gastric cancer cells by targeting ATG12 and HMGB2. Cell Death Dis 6: e1766, 2015.

17. Zhao L, Wang Y, Jiang L, He M, Bai X, Yu L and Wei M: MiR-302a/b/c/d cooperatively sensitizes breast cancer cells to adriamycin via suppressing $\mathrm{P}$-glycoprotein $(\mathrm{P}-\mathrm{gp})$ by targeting MAP/ERK kinase kinase 1 (MEKK1). J Exp Clin Cancer Res 35: 25,2016

18. Abraham I, Jain S, Wu CP, Khanfar MA, Kuang Y, Dai CL, Shi Z, Chen X, Fu L, Ambudkar SV, et al: Marine sponge-derived sipholane triterpenoids reverse P-glycoprotein (ABCB1)-mediated multidrug resistance in cancer cells. Biochem Pharmacol 80: 1497-1506, 2010.

19. Fayad W, Fryknäs M, Brnjic S, Olofsson MH, Larsson R and Linder S: Identification of a novel topoisomerase inhibitor effective in cells overexpressing drug efflux transporters. PLoS One 4: e7238, 2009.

20. Liu G, Pei F, Yang F, Li L, Amin AD, Liu S, Buchan JR and Cho WC: Role of autophagy and apoptosis in non-small-cell lung cancer. Int J Mol Sci 18: pii: E367, 2017.

21. Qian HR and Yang Y: Functional role of autophagy in gastric cancer. Oncotarget 7: 17641-17651, 2016.

22. Talaiezadeh A, Jalali F, Galehdari H and Khodadadi A: Time depended Bcl-2 inhibition might be useful for a targeted drug therapy. Cancer Cell Int 15: 105, 2015.

23. Cai Y, Xu P, Yang L, Xu K, Zhu J, Wu X, Jiang C, Yuan Q, Wang B, Li Y and Qiu Y: HMGB1-mediated autophagy decreases sensitivity to oxymatrine in SW982 human synovial sarcoma cells. Sci Rep 6: 37845, 2016. 
24. An X, Sarmiento C, Tan T and Zhu H: Regulation of multidrug resistance by microRNAs in anti-cancer therapy. Acta Pharm Sin B 7: 38-51, 2017.

25. O'Donovan TR, O'Sullivan GC and McKenna SL: Induction of autophagy by drug-resistant esophageal cancer cells promotes their survival and recovery following treatment with chemotherapeutics. Autophagy 7: 509-524, 2011.

26. Kumar A, Singh UK and Chaudhary A: Targeting autophagy to overcome drug resistance in cancer therapy. Future Med Chem 7: $1535-1542,2015$.

27. Li LQ, Xie WJ, Pan D, Chen H and Zhang L: Inhibition of autophagy by bafilomycin A1 promotes chemosensitivity of gastric cancer cells. Tumor Biol 37: 653-659, 2016.

28. Bhattacharya B, Low SH, Soh C, Kamal Mustapa N, Beloueche-Babari M, Koh KX, Loh J and Soong R: Increased drug resistance is associated with reduced glucose levels and an enhanced glycolysis phenotype. Br J Pharmacol 171: 3255-3267, 2014.
29. Yang $C$ and Pan Y: Fluorouracil induces autophagy-related gastric carcinoma cell death through Beclin-1 upregulation by miR-30 suppression. Tumour Biol: Jul 25, 2015 (Epub ahead of print).

30. Liu J, Zhang Y, Qu J, Xu L, Hou K, Zhang J, Qu X and Liu Y: $\beta$-Elemene-induced autophagy protects human gastric cancer cells from undergoing apoptosis. BMC Cancer 11: 183, 2011.

31. Mathew R, Karantza-Wadsworth V and White E: Role of autophagy in cancer. Nat Rev Cancer 7: 961-967, 2007.

32. Lee J, Giordano S and Zhang J: Autophagy, mitochondria and oxidative stress: Cross-talk and redox signalling. Biochem J 441: 523-540, 2012.

33. Zhu H, Wu H, Liu X, Li B, Chen Y, Ren X, Liu CG and Yang JM: Regulation of autophagy by a beclin 1-targeted microRNA, miR-30a, in cancer cells. Autophagy 5: 816-823, 2009. 